\title{
Zika virus: no cases in the Eastern Mediterranean Region but concerns remain
}

N.N. Tran Minh, ${ }^{7}$ Q. Huda, ${ }^{2}$ H. Asghar, ${ }^{3}$ D. Samhouri, ${ }^{4}$ A. Abubakar, ${ }^{5}$ C. Barwa,${ }^{6}$ I. Shaikh, ${ }^{7}$ E. Buliva, ${ }^{8}$ P. Mala ${ }^{9}$ and M. Malik ${ }^{10}$

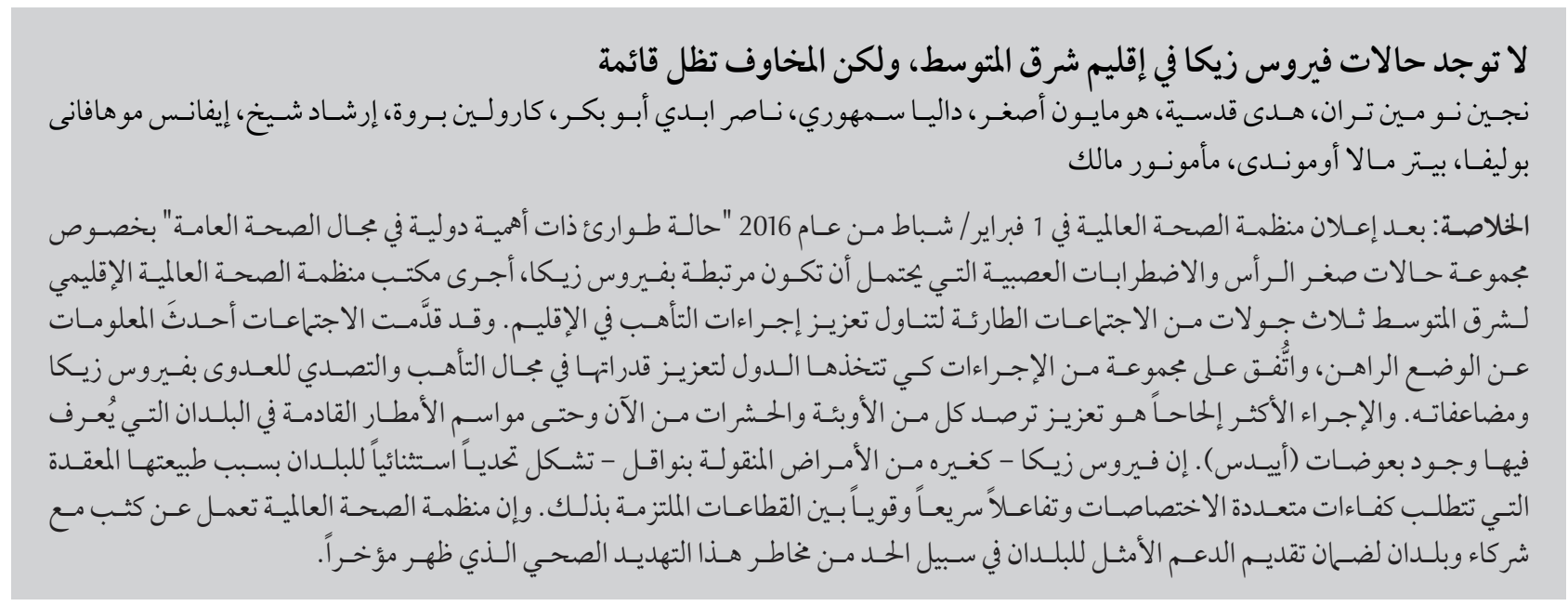

ABSTRACT Following the WHO declaration on 1 February 2016 of a Public Health Emergency of International Concern (PHEIC) with regard to clusters of microcephaly and neurological disorders potentially associated with Zika virus, the WHO Regional Office for the Eastern Mediterranean conducted three rounds of emergency meetings to address enhancing preparedness actions in the Region. The meetings provided up-to-date information on the current situation and agreed on a set of actions for the countries to undertake to enhance their preparedness and response capacities to Zika virus infection and its complications. The most urgent action is to enhance both epidemiological and entomological surveillance between now and the coming rainy seasons in countries with known presence of Aedes mosquitoes. Zika virus like other vector-borne diseases poses a particular challenge to the countries because of their complex nature which requires multidisciplinary competencies and strong rapid interaction among committed sectors. WHO is working closely with partners and countries to ensure the optimum support is provided to the countries to reduce the risk of this newly emerged health threat.

\begin{abstract}
Maladie à virus Zika : malgré l'absence de cas dans la Région de Méditerranée orientale les craintes persistent
RÉSUMÉ À la suite de la déclaration de l'OMS le 1er février 2016 faisant état d'une urgence de santé publique de portée internationale, eu égard à l'existence de groupes de cas de microcéphalie et de troubles neurologiques potentiellement associés à la maladie à virus Zika, le Bureau régional de l'OMS de la Méditerranée orientale a conduit trois cycles de réunions d'urgence en vue d'améliorer les mesures de préparation dans la Région. Les réunions ont fourni des informations actualisées de la situation actuelle et ont permis de convenir d'un ensemble d'actions à entreprendre par les pays afin d'améliorer leurs capacités de préparation et de réponse face à l'infection à virus Zika et ses complications. L'action la plus urgente consiste à améliorer la surveillance épidémiologique et entomologique à partir d'aujourd'hui et jusqu'à la prochaine saison des pluies dans les pays où la présence de moustiques Aedes est établie. La maladie à virus Zika, comme toutes les maladies à transmission vectorielle, constitue un défi pour les pays du fait de sa nature complexe qui nécessite des compétences multidisciplinaires et une interaction forte et rapide entre les secteurs impliqués.L'OMStravailleenétroitecollaborationavecsespartenairesetlespaysafindegarantirquelemeilleursoutiensoit apporté aux pays en vue de la réduction du risque de cette nouvelle menace sanitaire émergente.
\end{abstract}

'Epidemiologist, Pandemic and Epidemic Diseases, Department of Communicable Disease, WHO Regional Office for the Eastern Mediterranean, Cairo, Egypt (Correspondence to N.N. Tran Minh: tranminhn@who.int) (Authors' affilations continued on page 355)

Received: 15/03/16; accepted: 21/06/16 


\section{Introduction}

Since the Zika virus (ZIKV) was first identified in 1947 in Uganda (1), few human cases were reported until 2007 when the first Zika outbreak was reported in Yap, Micronesia (2). These early reports described the disease as relatively mild: about $20 \%$ of people infected with ZIKV develop mild fever, skin rash, conjunctivitis, arthralgia and/or arthritis, while the rest are asymptomatic. However, the virus became the latest threat to global health security when the Director-General of WHO declared on 1 February 2016 that recently reported clusters of microcephaly and neurological disorders potentially associated with ZIKV constituted a Public Health Emergency of International Concern (PHEIC) (3).

The temporal and geographic association between ZIKV infection and an increase in neurological disorders was first observed in French Polynesia in 2014 (4). During the current outbreak of ZIKV in America region, Brazil witnessed an apparent 20-fold increase in incidence of microcephaly cases from April 2014 to 2015 (5). In April 2016, a seminal review by scientists from the US Centers for Disease Control and Prevention concluded that a causal relationship exists between prenatal ZIKV infection and microcephaly and other serious brain anomalies (6). Among several studies reviewed were cases of vertical transmissions of ZIKV in women infected at the end of the first trimester of pregnancy, along with evidence of ZIKV in brain of two fetuses with microcephaly $(7,8)$. Based on these and other studies $(9,10)$, there is now scientific consensus that ZIKV is a cause of microcephaly and Guillain-Barré syndrome.

Following the declaration of the PHEIC, the WHO Regional Office for the Eastern Mediterranean conducted three rounds of emergency meetings with a view to enhancing preparedness and response capacities for ZIKV infection and its complications. The first two rounds of meetings held on 22-23 and
28-29 February in Cairo and Casablanca respectively had a good representation of the health ministries of the 22 Members States of the Region. These two meetings provided up-to-date information on ZIKV infection and its complications drew up a set of priority actions to be implemented by the Member States and WHO to enhance preparedness and response capacities. The third round of the meeting took place on 20-21 April 2016 in Cairo and called in regional partners including UN agencies, international organizations, academia and technical institutions to discuss the scope of scaling up the preparedness actions for Zika in the Region. The outcomes of the first two meetings with the Member States were discussed in the third meeting and a joint action plan was developed with partners to implement the priority actions in countries; this included a regionwide advocacy campaign to be launched jointly to raise awareness on Zika and other arboviral infections.

This overview highlights the current situation in the Region, the current knowledge gaps on the circulation of ZIKV in the past in the Region as well as recommended actions that the countries should consider to enhance their preparedness and response capacities.

\section{Situation in the EMR}

Although no human infection from ZIKV has so far been reported from any country in the Eastern Mediterranean Region, serological evidence of the circulation of ZIKV has been reported in at least two countries: Pakistan (11) and Egypt (12). The current situation does not rule out that cases of ZIKV infection may appear in the Region as travel is likely to contribute to the risk of importation of the virus. While the risk of importation is equally high in all countries in the Region, the risk of local transmission following the introduction of the virus through a viraemic patient returning from a country with active ZIKV circulation remains high in countries where the mosquitoes that primarily transmit this virus exist. According to this risk stratification, countries therefore need to enhance their preparedness measures.

Mosquitoes belonging the Aedes genus, particularly Aedes aegypti and Aedes albopictus, which are the species most incriminated as vectors of ZIKV and other arboviral diseases, have been found in a number of countries in the Region including Djibouti, Egypt, Lebanon, Oman, Pakistan, Saudi Arabia, Somalia, Sudan and Yemen. Sporadic cases of dengue fever, either locally transmitted or imported, have been reported from Djibouti, Egypt and Oman while explosive outbreaks of dengue fever have been reported in the past from Pakistan (13), Sudan (14) and Yemen (15), chikungunya from Yemen (16) and yellow fever from Sudan (17). These outbreaks have been propagated by the high density of Aedes mosquitoes that usually peaks during the summer months and also during the rainy season when water for household use is temporarily stored in household containers, especially in urban areas. It has been observed that temperature plays a role in adult vector survival, viral replication and infective period (18). These climatic conditions will favour the geographic expansion of the Aedes mosquitoes distribution and the risk of spread ofZIKV and other arboviral infections such as dengue, chikungunya and yellow fever in the endemic belt.

Vector-borne diseases pose a particular challenge to national public health authorities because of their complex nature requiring multidisciplinary competencies and strong rapid interaction among committed sectors. Aedes mosquitoes have been found in at least 9 countries of the Region, while their presence or absence is still to be assessed in the rest of the area. Therefore a strong entomological surveillance system is needed in the Region.

\section{Knowledge gaps on circulation of Zika virus in the Region}

A number of key knowledge gaps have been identified regarding the circulation or transmission of ZIKV in the past in the Region, including: a) Aedes 
vector distribution in the endemic belt; b) evidence of silent ZIKV circulation in the Region; c) evidence of increased incidence of microcephaly and GuillainBarré syndrome in the Aedes-endemic belt in the past during an active circulation of arboviruses; and d) causal link between microcephaly, Guillain-Barré syndrome and/or other neurological disorders and other arboviral infections in the endemic belt of Aedes mosquitoes during an active circulation of dengue, chikungunya and/ or yellow fever virus in the past.

\section{Public health measures for preventing ZIKV infection}

Mosquito surveillance remains as a key component of any local integrated vector management programme. Preventing or limiting the transmission of dengue, chikungunya and Zika viruses is completely dependent on the control of mosquito vectors and reduction of person-mosquito contact. A strong entomological surveillance system is crucial for obtaining data on the distribution of vector(s), the extent and types of breeding habitats, and the intensity and seasonal fluctuations of breeding of mosquitoes. Entomological sampling methods to assess Aedes population density and evaluate the control interventions have been largely implemented, especially in South-East Asian countries. There is a need to introduce and adapt those methods in countries through standardized protocols, including traditional Stegomyia indices to be used in outbreak prevention.

Lack of entomological capacity for arboviruses is one of the main contributing factors to poor entomological surveillance and control. An inventory of resources for medical entomology in the Region (i.e. experts, training institutes, research agencies and laboratories working for medical entomology) needs to be conducted. Furthermore, a network of medical entomologists needs to be established to support the work of entomological surveillance and vector control for arboviral diseases. A plan to build capacity for entomological surveillance and vector control through the use of regional/international resources/centres of expertise needs to be developed. The key vector control measures targeting all stages of the Aedes mosquitoes include environmental, biological and chemical methods. Population movement, climate change and insecticide resistance remain key concerns.

There is a strong need to build/ strengthen an early warning system to pre-empt/predict and detect early vectorborne diseases in the Region through integrating vector surveillance with syndromic based and event-based disease surveillance and using the surveillance data efficiently to predict and detect early the transmission risk. Operational research needs to be conducted to determine national thresholds for entomological indices that can be useful to pre-empt/ predict the occurrence of an epidemic.

The signs and symptoms of ZIKV infection often overlap with those of other arboviral infections making clinical diagnosis uncertain. Diagnosis can be confirmed by laboratory testing for the presence of ZIKV RNA or specific antiZika antibodies in the blood. However, a conventional antibody test (ELISA) in the Aedes-endemic belt remains a particular challenge because of cross-reactivity with other flaviviruses (e.g. dengue, yellow fever, West Nile viruses). The plaquereduction neutralization test (PRNT) for virus-specific neutralizing antibodies $(\operatorname{Ig} G)$ in serum samples is considered a confirmatory test. ZIKV may also present a risk to blood safety. There are reports of confirmed positive ZIKV RNA among healthy blood donors during the outbreak in French Polynesia (2013/14) (19) and recently two probable cases ofZIKV transmission by blood transfusion have been reported from Campinas, Brazil (20).

Countries reporting sporadic ZIKV in travellers arriving from affected countries pose little, if any, risk of onward transmission. Nonetheless, all travellers need to stay informed about ZIKV and other mosquito-borne diseases. They should adhere closely to steps that can prevent mosquito bites while travelling and practice safe sex, especially pregnant women and their partners, including through the correct and consistent use of condoms. Pregnant women need to be advised not to travel to areas of ongoing ZIKV transmission. Travellers to mass gatherings occurring in countries with active ZIKV transmission should follow the normal travel advice provided by their health authorities.

The International Health Regulations (IHR) 2005 requirements' at points of entry related to vector-borne diseases, as specified in Annex 5, call for establishing vector surveillance and control at points of entry and a minimum distance of $400 \mathrm{~m}$ from point of entry facilities (operations involving travellers, conveyances, containers, cargo and postal parcels). A vector surveillance and control programme should be appropriately designed for each point of entry using a risk assessment approach. Conveyances leaving a point of entry situated in an area where vector control is recommended should be disinfected. However, and in the context of ZIKV, the decision to implement WHO disinsection recommendations is dependent on individual country risk assessment for vector control. Currently, there is no WHO guiding document on disinsection of ships, however, the guidelines of the International Maritime Fumigation Organization on fumigating ships can be used as a reference (21).

Risk communication for ZIKV infection should take into consideration the varied context of countries in the Region, such as when a country is preparing for ZIKV, when a country has the first confirmed case of ZIKV with travel history to an affected country, when a country has the first confirmed case of ZIKV with no travel history, and when a country experiences a ZIKV outbreak. The public messages need to be timely, precise, applicable and relevant, i.e. tailored to the audience for which they are intended. Communication products should be tested (pre and post) to analyse the public impact of their messages and to ascertain whether behavioural changes to control the vector have in fact been achieved. 
In view of the extensive actions that need to be taken forward in the countries in all the areas of work outlined above, the Member States acknowledged the importance of having strong public health leadership for effective coordination among all stakeholders, including the community, to reduce the risk of ZIKV infection in countries.

\section{Conclusion}

During the current ongoing outbreak, WHO estimates that there could be 3-4 million cases of ZIKV infection in the Americas in the next 12 months (22). The risk of international spread of ZIKV to other regions is a real concern, as demonstrated by the recent finding that the Zika strain responsible for the outbreak in Cape Verde, Africa was most likely imported from Brazil (23). In the EMR, the risk of local transmission poses further concern. Owing to a lack of laboratory diagnostic capacities for ZIKV in countries where the Aedes mosquitoes exist, many of which are affected by protracted conflict and have fragile health systems, any silent introduction of the virus may go undetected and trigger sustained local transmission. As populations living in the countries in the Aedes mosquito belt would supposedly be immunologically naïve to ZIKV, the ubiquitous presence of the vectors might contribute to rapid transmission of ZIKV amongst the at-risk population if not detected and contained early.

The current situation where no cases ofZIKV have been reported in the Region, either locally acquired or travelassociated, presents an opportunity to improve surveillance and fill other gaps in preparedness and response capacities as required under the IHR 2005. Boxes 1 and 2 shows a number of recommendations that countries may consider to implement; these came out of the two rounds of meetings in February 2016. The most urgent is to enhance both epidemiological and entomological surveillance between now and the coming rainy seasons in countries with a known presence of Aedes mosquitoes. Such efforts should be directed to both early identification and recognition of ZIKV transmission as well as identification of any abnormal or sudden increase in congenital birth defects for which there is no plausible reason. As the density of Aedes mosquitoes is expected to peak during the summer months and during the rainy seasons in these high-risk countries, necessary vector control efforts need to be directed at reducing the potential breeding sites of mosquito populations and/or their interactions with humans to below levels that can sustain an epidemic.

Such actions certainly warrant heightened support from all concerned. WHO is working closely with partners from the countries and other regions, as well as other UN agencies, in order to ensure optimum support to countries to reduce the risk ofZIKV infection and its complications.

The countries of the EMR demonstrated outstanding solidarity, public health vigilance and shared responsibilities during the threat of introduction of Ebola virus disease. The time has again come to show the same level of collective understanding, purpose and effort in order to mitigate this newly emerging health threat.

\section{References}

1. Musso D, Cao-Lormeau VM, Gubler DJ. Zika virus: following the path of dengue and chikungunya? Lancet. $2015 \mathrm{Jul}$ 18;386(9990):243-4. PMID:26194519

2. Duffy MR, Chen TH, Hancock WT, Powers AM, Kool JL, Lanciotti RS, et al. Zika virus outbreak on Yap Island, Federated States of Micronesia. N Engl J Med. 2009 Jun 11;360(24):2536-43. PMID:19516034

3. WHO statement on the first meeting of the International Health Regulations (2005) (IHR 2005) Emergency Committee on Zika virus and observed increase in neurological disorders and neonatal malformations. February 1, 2016 (http://www.who.int/ mediacentre/news/statements/2016/1st-emergency-committee-zika/en/, accessed 3 May 2016).

4. Ioos S, Mallet HP, Leparc Goffart I, Gauthier V, Cardoso T, Herida M. Current Zika virus epidemiology and recent epidemics. Med Mal Infect. 2014 Jul;44(7):302-7. PMID:25001879

5. Fauci AS, Morens DM. Zika Virus in the Americas-Yet Another Arbovirus Threat. N EngI J Med. 2016 Feb 18;374(7):601-4. 10.1056/NEJMp1600297 PMID:26761185

6. Rasmussen SA, Jamieson DJ, Honein MA, Petersen LR. Zika Virus and Birth Defects-Reviewing the Evidence for Causality. N Engl J Med. 2016 May 19;374(20):1981-7. PMID:27074377

7. Mlakar J, Korva M, Tul N, Popović M, Poljšak-Prijatelj M, Mraz J, et al. Zika Virus Associated with Microcephaly. N Engl J Med. 2016 Mar 10;374(10):951-8. PMID:26862926
8. Driggers RW, Ho CY, Korhonen EM, Kuivanen S, Jääskeläinen AJ, Smura T, et al. Zika Virus Infection with Prolonged Maternal Viremia and Fetal Brain Abnormalities. N Engl J Med. 2016 Jun 2;374(22):2142-51. 10.1056/NEJMoa1601824 PMID:27028667

9. Cao-Lormeau V-M, Blake A, Mons S, Lastère S, Roche C, Vanhomwegen J, et al. Guillain-Barré Syndrome outbreak associated with Zika virus infection in French Polynesia: a case-control study. Lancet. 2016 Apr 9;387(10027):1531-9. 10.1016/S01406736(16)00562-6 PMID:26948433

10. Cauchemez S, Besnard M, Bompard P, Dub T, GuillemetteArtur P, Eyrolle-Guignot D, et al. Association between Zika virus and microcephaly in French Polynesia, 2013-15: a retrospective study. Lancet. 2016 May 21;387(10033):2125-32. 10.1016/S01406736(16)00651-6 PMID:26993883

11. Darwish MA, Hoogstraal H, Roberts TJ, Ahmed IP, Omar F. A seroepidemiological survey for certain arboviruses (Togaviridae) in Pakistan. Trans R Soc Trop Med Hyg. 1983;77(4):442-5.

12. Hayes EB. Zika virus outside Africa. Emerg Infect Dis. 2009 Sep;15(9):1347-50. PMID:19788800

13. Haider Z, Ahmad FZ, Mahmood A, Waseem T, Shafiq I, Raza T, et al. Dengue fever in Pakistan: a paradigm shift; changing epidemiology and clinical patterns. Perspect Public Health. 2015 Nov;135(6):294-8. 10.1177/1757913915599019 PMID:26342006

14. Seidahmed OM, Siam HA, Soghaier MA, Abubakr M, Osman HA, Abd Elrhman LS, et al. Dengue vector control and surveil- 
lance during a major outbreak in a coastal Red Sea area in Sudan. East Mediterr Health J. 2012 Dec;18(12):1217-24. PMID:23301396

15. Bin Ghouth AS, Amarasinghe A, Letson GW. Dengue outbreak in Hadramout, Yemen, 2010: an epidemiological perspective. Am J Trop Med Hyg. 2012 Jun;86(6):1072-6. 10.4269/ ajtmh.2012.11-0723 PMID:22665621

16. Malik MR, Mnzava A, Mohareb E, Zayed A, Al Kohlani A, Thabet AA, et al. Chikungunya outbreak in Al-Hudaydah, Yemen, 2011: epidemiological characterization and key lessons learned for early detection and control. J Epidemiol Glob Health. 2014 Sep;4(3):203-11. 10.1016/j.jegh.2014.01.004 PMID:25107656

17. Markoff L. Yellow fever outbreak in Sudan. N Engl J Med. 2013 Feb 21;368(8):689-91. 10.1056/NEJMp1300772 PMID:23387798

18. Morin CW, Comrie AC, Ernst K. Climate and dengue transmission: evidence and implications. Environ Health Perspect. 2013 Nov-Dec;121(11-12):1264-72. PMID:24058050

19. Aubry M, Finke J, Teissier A, Roche C, Broult J, Paulous S, et al. Seroprevalence of arboviruses among blood donors in French
Polynesia, 2011-2013. Int J Infect Dis. 2015 Dec;41:11-2. 10.1016/j. ijid.2015.10.005 PMID:26482390

20. CIDRAP - Center for Infectious Disease Research and Policy. Brazil confirms blood-transfusion Zika; PAHO calls for global support (http://www.cidrap.umn.edu/news-perspective/2016/02/brazil-confirms-blood-transfusion-zika-pahocalls-global-support, accessed 3 May 2016).

21. IMFO - International Maritime Fumigation Organisation. Code of Practice on Safety and Efficacy for Marine Fumigation, 2010 (http://www.imfo.com/IMFO_Code_of_Practice.pdf, accessed 3 May 2016).

22. Samarasekera U, Triunfol M. Concern over Zika virus grips the world. Lancet. 2016 Feb 6;387(10018):521-4. 10.1016/S01406736(16)00257-9 PMID:26852261

23. WHO confirms Zika virus strain imported from the Americas to Cabo Verde. May 20, 2016 (http://who.int/mediacentre/ news/releases/2016/zika-cabo-verde/en/, accessed 3 May 2016)

\section{Box 1 Recommended actions to enhance preparedness and response capacities for prevention and control of Zika virus infection in EMR countries with evidence of transmission of dengue, chikungunya and yellow fever viruses in the past}

1. Identify hot spots (risk mapping) through uniform and standardized risk assessment and stratify areas by spatial and geographic distribution of Aedes mosquitoes and past arbovirus epidemics.

2. Develop/update a geo-referenced atlas of Aedes and their current susceptibility status, which should be used as the basis for developing an insecticide resistance management strategy; use this information to update the categorization of high risk, low risk and receptive countries.

3. Establish a sentinel surveillance system for Aedes mosquitoes in areas with high density including at designated points of entry, and collect data regularly to pre-empt and detect the occurrence of high densities of Aedes mosquitoes and target these areas for vector control.

4. Develop/update an integrated vector management strategy with strong focus on entomological surveillance and control measures, supported by a clear operational plan defining the role of each sector.

5. Establish an early warning system for detection of clusters of ZIKV infection and other vector-borne diseases by:

- using a uniform case definition for syndromic surveillance of acute febrile syndrome;

- using a sentinel-based syndromic surveillance system for clusters of acute febrile syndromes in high risk areas (hot spots) as complementary to the routine disease surveillance system;

- integrating the syndromic disease surveillance system with the sentinel based entomological surveillance system for Aedes mosquitoes and periodically share disease and entomological surveillance data and other information;

- establishing an event-based surveillance system (including a community-based surveillance system where possible)

- utilizing existing surveillance (e.g. measles, birth defects and acute flaccid paralysis) to detect ZIKV infection and its complications;

- enhancing timely reporting and information sharing between the epidemiology, laboratory and entomology surveillance units.

6. Improve appropriate laboratory diagnosis and testing capacities for ZIKV infection, including establishing appropriate links with external reference laboratories for sample testing.

7. Keep blood transfusion services updated about measures to ensure a safe supply of blood.

8. Consider disinfecting conveyances arriving from a country with active ZIKV transmission using risk assessment approach and using WHO standard recommendations for disinsection.

9. Establish a sentinel based surveillance system for congenital birth defects using consistent case definitions and harmonized data collection tools, and follow-up clinical outcomes of pregnant women suspected to be infected with an arboviral disease.

10. Collect retrospective data on birth registrations, trace them back and try to establish any baseline/comparator data to assess the trend of congenital birth defects (especially microcephaly).

11. Conduct regular public awareness campaigns to proactively inform the public of the ZIKV situation, urge communities to keep areas in and around the home free from mosquito breeding sites through applying appropriate risk communication messages and strategies in accordance with local culture and behavioural practice, and provide communities with the appropriate knowledge, information and tools to protect themselves from mosquito bites.

12. Develop/update epidemic and pandemic contingency plans for ZIKV infection as well as standard operating procedures for an incident command system and for strengthening coordination. 
Box 2 Recommended actions to enhance preparedness and response capacities for prevention and control of Zika virus infection in EMR countries with no presence of Aedes mosquitoes or with presence of Aedes mosquitoes but no evidence of transmission of dengue, chikungunya and yellow fever viruses in the past

1. Raise awareness of travellers going to areas with active transmission, including those participating in mass gathering activities, through issuance of appropriate advisories to reduce the possibility of exposure to mosquito bites.

2. Enhance coordination and collaboration between travel, trade and health sectors to ensure implementation of related WHO recommendations.

3. Increase knowledge and awareness of all aspects of ZIKV infection among clinicians, health care workers and other stakeholders.

4. Improve appropriate laboratory diagnosis and testing capacities for ZIKV infection, including establishing appropriate links with external reference laboratories for sample processing.

5. Keep blood transfusion services updated about measures to ensure a safe supply of blood.

6. Establish sentinel surveillance of Aedes mosquitoes in areas with past information of Aedes distribution and take prompt action targeting breeding sites in a radius of $400 \mathrm{~m}$ in the event of any increase in Aedes density.

7. Conduct surveys to collect data on Aedes mosquitoes in countries with no known vectors and enhance surveillance of mosquitoes in areas bordering countries with known vectors.

8. Monitor imported goods (e.g. used tyres, bamboo) by quarantine measures to avoid entry of invasive species of mosquitoes and implement vector surveillance and control at designated points of entry as per IHR (2005).

${ }^{2}$ Regional Adviser, Health Risk Management; ${ }^{3}$ Regional Adviser, Public Health Laboratories; ${ }^{4}$ Technical Officer, Epidemiological Surveillance and International Health Regulations; ${ }^{5}$ Medical Officer, Pandemic and Epidemic Diseases; ${ }^{7}$ Former Regional Adviser, Epidemiological Surveillance and International Health Regulations; ${ }^{8}$ Consultant, Pandemic and Epidemic Diseases; ${ }^{9}$ Medical Officer, Pandemic and Epidemic Diseases, Health Security and Regulations, Department of Communicable Disease, WHO Regional Office for the Eastern Mediterranean, Cairo, Egypt. ${ }^{6}$ Technical Officer, Malaria Control and Elimination, Department of Communicable Disease, WHO Regional Office for the Eastern Mediterranean, Cairo, Egypt. ${ }^{10}$ Coordinator, Health Security and Regulations, Department of Communicable Disease, WHO Regional Office for the Eastern Mediterranean, Cairo, Egypt. 